\title{
Post-operative antibiotic usage at Benghazi Medical Center, Libya between 2009 and 2012
}

\author{
Nouh M H. Aljarari ${ }^{1}$, Jagannadha Rao Peela ${ }^{2}$ \\ 1. Department of Pharmacology, Faculty of Medicine, Benghazi University, Benghazi-Libya \\ 2. Department of Biochemistry, Faculty of Medicine, Quest International University Perak, 30250 Ipoh, \\ Malaysia
}

\section{Introduction}

Surgical site infections are the most common hospital-acquired infections among surgical patients, ${ }^{1}$ and are frequent and serious complications of surgical procedures. ${ }^{2}$ According to the United States Canters for Disease Control (CDC) National Nosocomial Infection Surveillance (NNIS) system, 14-16\% of hospitalacquired infections were contributed by surgical site infections and were the third most common hospitalacquired infections. ${ }^{3}$

Surgical site infections are associated with increased antibiotic usage, increased costs, ${ }^{4}$ prolonged hospitalization, readmissions, re-interventions, permanent disability or even death of the patient. ${ }^{1}$ Although adequate antibiotic prophylaxis can reduce the risk of surgical site infections, this favours the emergence of antimicrobial resistance. Surgical antibiotic prophylaxis (SAP) is defined as the usage of antibiotics to prevent infections at the surgical site. About $30-50 \%$ of antibiotic use in hospitals is for surgical prophylaxis; however $30-90 \%$ of this prophylaxis is not appropriate. ${ }^{4}$ The major challenges associated with correct SAP are the indications, type of procedure, the antibiotic prescribed, dosage and duration of the treatment. The major problem areas are the selection of appropriate antibiotic, timing, and dosage formula, route of administration and duration of SAP. ${ }^{3}$

There are several factors influencing the antibiotic prophylaxis prescribed by a surgeon including lack of awareness of available guidelines, lack of adherence to these guidelines, personal choices, influence from medical school training and colleagues and lack of implementation of antibiotic policy in the hospital. ${ }^{3}$ Lack of proper microbiology facilities and unwillingness of patients to undergo culture-sensitivity tests all are additional factors. ${ }^{5}$

All these aspects add to the problem of antibiotic misuse, resulting in bacterial resistance, which is a serious threat to patient's health. Additionally, very few novel antibiotics are currently being developed; ${ }^{6,7}$ because of which the only way resistance can be delayed is by an appropriate and frugal use of this group of drugs. $^{7}$

We conducted the present study to analyze the pattern of postoperative antibiotic utilization in the years 20092012, both as monotherapy and/or as combination therapy at Benghazi Medical Center (BMC), Libya. We also aimed at uncovering the reasons of antibacterial drug abuse and adopt appropriate strategies to circumvent this problem.

\section{Materials and Methods}

This was a retrospective study, where in data on antibiotic prescriptions both as monotherapy and as combination therapy, between 2009 and 2012 in the postoperative wards of the Benghazi Medical Center (BMC), Libya was retrieved from the hospital records.

The various parameters assessed were date of the prescription, age and gender of the patients, whether in-patient or out-patient, results of the culture sensitivity test, antibiotic/s prescribed, the dose, duration and condition for which they were prescribed.

\section{Results}

In the present study, records of 1379 patients admitted in the postoperative wards during the years 2009-2012 were included. The age and year-wise distribution is shown in fig. 1. The gender-wise distribution is shown in fig. 2. 

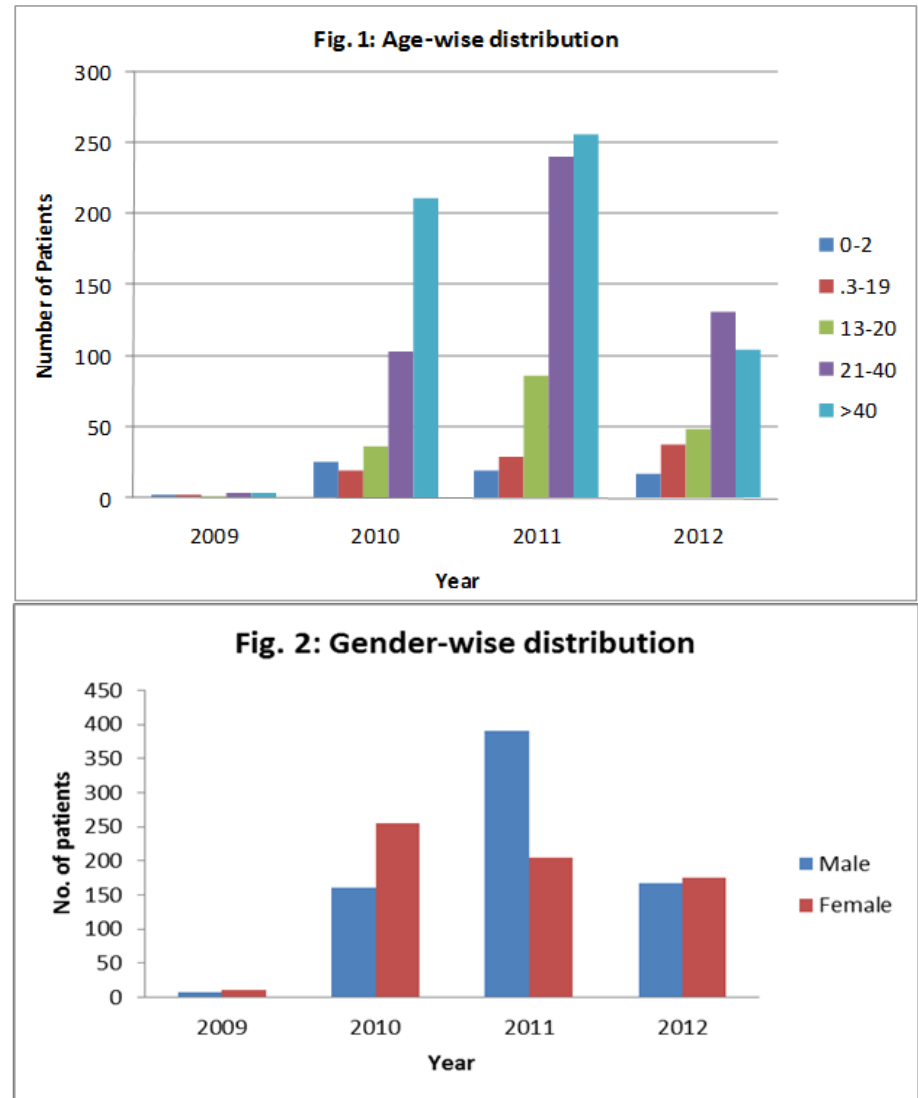

Ciprofloxacin, ceftriaxone and amoxicillin/clavulanic acid were the most frequently prescribed antibiotics according to our study.

The number of patients receiving each of these drugs is described in table 1.

\begin{tabular}{|c|c|c|c|c|c|c|}
\hline & \multicolumn{2}{|c|}{ Ciprofloxacin } & \multicolumn{2}{c|}{ Ceftrioxone } & \multicolumn{2}{c|}{ Amoxicillin/Clavulanic acid } \\
\hline year & male & female & male & female & male & female \\
\hline 2009 & 1 & 1 & 3 & 4 & 1 & 4 \\
\hline 2010 & 16 & 22 & 47 & 130 & 45 & 57 \\
\hline 2011 & 19 & 28 & 82 & 55 & 142 & 51 \\
\hline 2012 & 18 & 9 & 31 & 47 & 63 & 66 \\
\hline
\end{tabular}

The details of the various antibiotics combinations used are described in table 2.

\begin{tabular}{|l|l|l|}
\hline Table 2: Antibiotic combinations prescribed \\
\hline Year & Antibiotic combination & Number of patients \\
\hline 2009 & Vancomycin + ciprofloxacin & 1 \\
& Amoxicillin/clavulanic acid + ceftriaxone & 1 \\
\hline 2010 & Ceftriaxone+cefalexin & 12 \\
& Ampicillin+cloxacillin & 8 \\
& Ceftriaxone+ciprofloxacin & 6 \\
\hline 2011 & Ceftriaxone + gentamicin & 58 \\
& Amoxicillin/clavulanic acid + Ceftriaxone & 37 \\
& Amoxicillin/clavulanic acid + ciprofloxacin & 8 \\
\hline 2012 & Ceftriaxone + clarithromycin & 2 \\
& Ceftriaxone + clarithromycin & 1 \\
& Ceftriaxone+ suprax & 1 \\
\hline
\end{tabular}

\section{Discussion}

In our study, we found that ceftriaxone, amoxicillin/clavulanic acid and ciprofloxacin were the most frequently prescribed drugs for the prevention of postoperative infections during the years 2009-2012. The most common cases were the patients undergoing surgery for cholelithiasis.

Ceftriaxone was the most frequently prescribed antibiotic in our hospital, the reason being easy availability of the drug in the hospital pharmacy. From the years 2009, 2010 and 2012, it has been observed that 
more women were prescribed this drug compared to men. The next most common drug prescribed was a combination of amoxicillin and clavulanic acid, which was more commonly prescribed in men from the years 2009, 2010 and 2012, in contrast to ceftriaxone. Ciprofloxacin was third most frequently prescribed antibiotic in our hospital. More number of females received the drug across all the four study years, unlike the case of the previous two drugs.

Additionally, as seen in table 1, the data from patients was substantially less in 2009 , probably because at the time, the hospital had just started. Another interesting observation made was that the antibiotic prescriptions were lesser in 2012 compared to the years 2010 and 2011. This variation could be attributed to the political and security situation of the country. It could also be due to increasing awareness of the surgeons' indiscriminate prescription of these drugs.

Several studies have already shown that broad-spectrum antibiotics are often indiscriminately prescribed. ${ }^{8-10}$ In a survey in a teaching hospital in Ethiopia to determine the prescribing practices, the most commonly prescribed forms of antibiotics were amoxicillin (16.4\%), ampicillin $(15 \%)$, gentamicin $(14.9 \%)$ and chloramphenicol (11.6\%). However, the most commonly prescribed injectable antibiotics were ampicillin $(21.4 \%)$, cloxacillin $(13.4 \%)$, crystalline penicillin $(12.4 \%)$, ceftriaxone $(9.8 \%)$, gentamicin $(9.8 \%)$ and chloramphenicol $41(8.4 \%){ }^{11}$

In a study by Bhansali et al., most commonly prescribed antibiotics were cefixime tablets $(64.44 \%)$, and ceftriaxone $(44.03 \%)$ and amikacin injectables $(36.67 \%){ }^{12}$ In another study by Salman et al., similar to our study, injection ceftriaxone was the most common drug prescribed in postoperative patients. Injection Amikacin was also frequently prescribed. ${ }^{13}$

In our study, some combination antibiotics were also prescribed as seen in table 2. Ceftriaxone with gentamicin was the commonest combination, followed by ceftriaxone with amoxicillin/clavulanic acid. However, the use of antibiotics in combination is often irrational and without any scientific evidence. ${ }^{12}$

Whenever possible it is advisable to prescribe an antibiotic with the narrowest antibacterial spectrum, based on the culture/sensitivity reports to reduce the emergence of multi-drug resistant pathogens. ${ }^{4}$ In our study, culture/sensitivity tests were not performed, and the antibiotics were prescribed based on observation. Ideally, broad-spectrum antibiotics like third generation cephalosporins (e.g., ceftriaxone and cefotaxime) should be avoided in surgical prophylaxis. However, in our study as well as in the other studies discussed, we observe that these drugs have been the most frequently prescribed antibiotics. ${ }^{4}$

In our hospital, antibiotic misuse is probably due to the factors shown in box 1.

\section{Box 1: Reasons for antibiotic misuse at BMC}

- The routine use of antibiotics for almost all patients post-operatively

- Empirical therapy which is not based on culture and sensitivity results as evidence.

- Improper timing and duration of prophylactic post-operative use of antibiotics.

- Poor sanitation measures in both hospital wards and operation theatres which may be the reason for post-operative wound infections

These issues could be addressed by taking measures as described in box 2 .

\section{Box 2: Measures to overcome the problem of antibiotic misuse}

- Antibiotic prescription should be based on the clinical condition supported by the results of culture and sensitivity tests.

- Patients should be instructed properly based on their educational level and create awareness about their disease condition.

- Patients should be instructed properly about the duration of the antibiotic usage create awareness about the need to follow the entire course of treatment.

- Private pharmacies should not dispense antibiotics without prescription from the treating physician.

\section{Conclusion}

Over prescription of antibiotics by the clinicians and it's inappropriate use is still a common practice in many hospitals in developing countries and our hospital is no exception. Prescription of broad spectrum drugs empirically, without evidenced by culture and sensitivity tests is also a major problem encountered, which is compounded by poor patient compliance. All these practices result in emergence of multiple antibiotic resistant strains of bacteria. Therefore, there is a need for strict antibiotic prescription policies in our hospital, which should be developed by a collaborative effort involving physicians, surgeons and microbiologists. Paramedical professionals should be adequately trained, in order to ensure proper implementation of these policies. Lastly, the role of adequate information dissemination to the patients cannot be undermined. 
In conclusion BMC being a teaching hospital and tertiary care centre has major responsibility to curb irrational drug prescription by the doctors and there is need to follow ethical guidelines on a proper prescription of drugs and to ensure that the patients utilize drugs only on prescription and in right formulations. The support staffs in the hospital also need to take responsibility for proper implementation of the prescription policies in disease management and prophylaxis.

\section{Acknowledgements:}

This is my sincere thanks to my students Bassema M. Saad, Basma A. Elmansoury, Hanan H. Salem \& Hiba M. Issa, Faculty of Pharmacy, Benghazi University to collect data from the hospital (BMC) wards. I am very much thankful to Dr.Shakila Srikumar, Biochemistry, Dr.Soe Nung, Community Medicine, Faculty of Medicine, Quest International University Perak, Ipoh, Malaysia and Dr.B.R.Ambhetkar Medical College, Bengaluru, India for their support to give final shape to this manuscript.

\section{References}

[1]. Perencevich EN, Sands KE, Cosgrove SE, Guadagnoli E, Meara E, et al. Health and economic impact of surgical site infections diagnosed after hospital discharge. Emerg Infect Dis. 2003;9:196-203.

[2]. Crolla RM, van der Laan L, Veen EJ, et al. Reduction of surgical site infections after implementation of a bundle of care. PLoS One. 2012;7(9):e44599.

[3]. Ng RS, Chong CP. Surgeons' adherence to guidelines for surgical antimicrobial prophylaxis - a review. Australas Med J. 2012;5(10):534-40.

[4]. Munckhof W. Antibiotics for surgical prophylaxis. Aust Prescr. 2005;28:38-40

[5]. Kotwani A, Wattal C, Katewa S, et al. Factors influencing primary care physicians to prescribe antibiotics in Delhi India. Fam Pract. 2010;27(6):684-90.

[6]. Barlow G, Nathwani D. Is antibiotic resistance a problem? A practical guide for hospital clinicians. Postgrad Med J 2005;81:680692.

[7]. Pulcini C, Gyssens IC. How to educate prescribers in antimicrobial stewardship practices. Virulence. 2013;4(2):192-202.

[8]. Ochoa C, Eiros JM, Inglada L, Vallano A, Guerra L. Assessment of antibiotic prescription in acute respiratory infections in adults. The Spanish Study Group on Antibiotic Treatments. J Infect. 2000;41:73-83.

[9]. Piccirillo JF, Mager DE, Frisse ME, Brophy RH, Goggin A. Impact of first-line vs second-line antibiotics for the treatment of acute uncomplicated sinusitis JAMA. 2001;286:1849-56.

[10]. Salvo F, Polimeni G, Moretti U, Conforti A, Leone R, Leoni O, et al. Adverse drug reactions related to amoxicillin alone and in association with clavulanic acid: data from spontaneous reporting in Italy. J Antimicrob Chemother. 2007;60:121 -26.

[11]. Desalegn AA. Assessment of drug use pattern using WHO prescribing indicators at Hawassa University Teaching and Referral Hospital, south Ethiopia: a cross-sectional study. BMC Health Serv Res. 2013;13:170.

[12]. Bhansali NB, Gosai TR, Dholaria NK, et al. Drug utilization study in post-operative patients in surgical ward of a tertiary hospital attached with medical college. Der Pharmacia Lettre. 2013;5(1):251-257.

[13]. Salman MT, Akram MF, Rahman S, et al. Drug prescribing pattern in surgical ward of a teaching hospital in North India. Ind J Pract Doctor. 2008;5(2):5-6. 